\title{
Images in Clinical Tropical Medicine Motile Onchocerca volvulus Microfilariae in the Anterior Chamber of the Eye
}

\author{
Michael E. Gyasi, ${ }^{1}$ Augustine R. Hong, ${ }^{2}$ and Gary J. Weil ${ }^{3 *}$ \\ ${ }^{1}$ St. Thomas Eye Hospital, Accra, Ghana; ${ }^{2}$ Department of Ophthalmology, Washington University School of Medicine, St. Louis, Missouri; \\ ${ }^{3}$ Infectious Diseases Division, Department of Medicine, Washington University School of Medicine, St. Louis, Missouri
}

A 59-year-old man from an onchocerciasis-endemic area in eastern Ghana was enrolled in a clinical trial to assess the impact of ivermectin on microfilariae (Mf) in the skin and eyes. He had palpable subcutaneous nodules consistent with onchocerciasis and

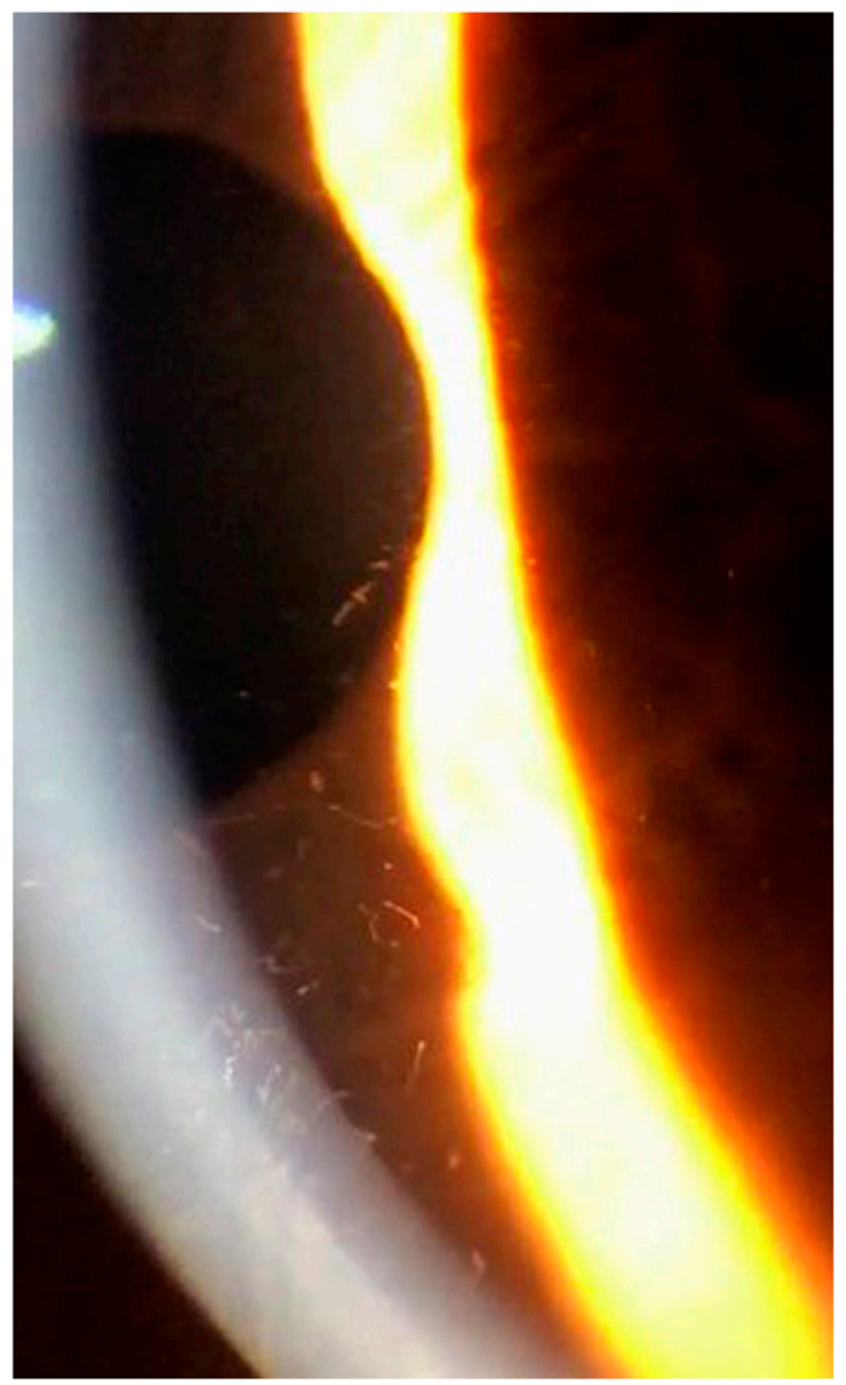

FIGURE 1. This screenshot from the slit lamp video shows Onchocerca volvulus microfilariae (Mf) in the anterior chamber of the eye. Some Mf overlie the pupil (in the upper left of the image). This figure appears in color at www.ajtmh.org. a high Mf burden in skin snips (170/mg of skin). He reported mild generalized pruritus and blurry vision in both eyes. Visual acuity was 20/80 (OD) and 20/60 (OS). Both eyes had moderate cataracts and features consistent with prior anterior uveitis. Intraocular pressures were normal (12 mmHg OU). Atrophic changes in his optic nerves correlated well with visual field defects measured by frequency doubling technology and with retinal nerve fiber layer loss as assessed by optical coherence tomography. Slit lamp examination revealed high numbers of motile $\mathrm{Mf}$ in the anterior chambers of both eyes (100-OD and 50-OS) (Figure 1). A cell-phone video (Supplemental Video) illustrates the dramatic appearance of Mf in the anterior chamber by a slit lamp.

Ocular Mf were still present but decreased in numbers and motility (40-OD and 20-OS) 3 months after ivermectin treatment (150 mg/kg); no Mf were detected in four skin snips taken from each iliac crest and calf at that time. Microfilariae were still absent in skin snips taken 6 months after treatment when ocular Mf counts were 20-OD and 15-OS.

Onchocerca volvulus Mf were cleared from the eyes more slowly than from the skin after ivermectin treatment. The patient's vision did not improve after treatment presumably because of irreversible effects of onchocerciasis.

Received January 10, 2020. Accepted for publication January 15, 2020.

Note: Supplemental video appears at www.ajtmh.org.

Acknowledgments: We would like to acknowledge contributions and leadership by Nicholas Opoku, the principal investigator for the clinical trial who enrolled this participant. We also would like to thank the ophthalmology research team at the University of Health and Allied Sciences School of Public Health in Hohoe, Ghana.

Financial support: This work was supported by grant OPP GH5340 from the Bill \& Melinda Gates Foundation.

Disclaimer: The funder had no role in the planning or performance of this study, writing, or the decision to publish this paper.

Authors' addresses: Michael E. Gyasi, St. Thomas Eye Hospital, Accra, Ghana, E-mail: mikegyasi67@gmail.com. Augustine R. Hong, Department of Ophthalmology, Washington University School of Medicine, St. Louis, MO, E-mail: ahong22@wustl.edu. Gary J. Weil, Infectious Diseases Division, Department of Medicine, Washington University School of Medicine, St. Louis, MO, E-mail: gary.j.weil@ wustl.edu.

This is an open-access article distributed under the terms of the Creative Commons Attribution (CC-BY) License, which permits unrestricted use, distribution, and reproduction in any medium, provided the original author and source are credited.

\footnotetext{
*Address correspondence to address Gary J. Weil, Infectious Diseases Division, Washington University School of Medicine, Box 8051, 660 S. Euclid Ave., St. Louis, MO 63110. E-mail: gary.j.weil@wustl.edu
} 